\title{
Efficacy and Safety of Aflibercept and Its Role in the Treatment of Metastatic Colorectal Cancer
}

\author{
Mohamed E. Salem • Sherif M. El-Refai
}

To view enhanced content go to www.rarecancers-open.com Received: July 23, 2013 / Published online: October 16, 2013

(C) The Author(s) 2013. This article is published with open access at Springerlink.com

\begin{abstract}
Background: Vascular endothelial growth factor (VEGF) and related pathway elements are critically important in the pathogenesis, growth, and development of solid tumors. Inhibiting the VEGF signaling pathway is being investigated as a therapeutic strategy for cancer, either by blockade of VEGF ligand binding or inhibition of the VEGF receptor. Aflibercept is a VEGF inhibitor that has been approved by the US Food and Drug Administration for the treatment of macular degeneration and metastatic colorectal cancer (mCRC).
\end{abstract}

M. E. Salem (ه)

Karmanos Cancer Institute, Wayne State University, 4100 John R, HW04H0, Detroit, MI 48201, USA e-mail: Salemm@karmanos.org

S. M. El-Refai

University of Florida, Gainesville, FL, USA

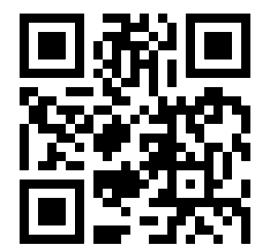

Enhanced content for this article is available on the journal web site: www.rarecancers-open.com
Methods: A search of PubMed and MEDLINE from January 2000 to August 2013 was performed using the following terms (or combination of terms): VEGF, colorectal cancer, mCRC, aflibercept, ziv-aflibercept. Studies were limited to those published in English.

Results: Phase 1 and 2 clinical trials of aflibercept in advanced non-hematological cancer showed acceptable safety and provided justification for further investigation. Four phase 3 clinical trials were conducted to evaluate aflibercept for the treatment of different cancers. The VELOUR trial evaluated aflibercept with FOLFIRI in patients with mCRC. The addition of aflibercept resulted in improved median overall survival (OS, 13.5 vs. 12.1 months, $p=0.0032)$. The VITAL trial evaluated docetaxel plus aflibercept vs. docetaxel plus placebo in patients with nonsmall cell lung cancer (NSCLC) who had progressed after first-line platinum-based chemotherapy; there was no significant difference between the two treatment groups in terms of OS. The VANILLA trial in patients with metastatic pancreatic adenocarcinoma was terminated because addition of aflibercept to 
gemcitabine failed to demonstrate improved OS in an interim analysis. In patients with metastatic androgen-independent prostate cancer, the VENICE trial also failed to demonstrate an increase in OS when aflibercept was combined with standard firstline chemotherapy. The most common adverse events associated with aflibercept in these trials were hypertension, venous and arterial thromboembolic events, hemorrhage, and proteinuria.

Conclusions: In patients with mCRC, aflibercept demonstrated an improvement in OS when added to FOLFIRI. However, it has failed to show similar benefits in other tumor types. Further investigation is needed to improve patient selection and to distinguish patients who may benefit best from aflibercept from those who will require other antiangiogenic agents.

Keywords: Adverse events; Aflibercept; Colorectal cancer; Tumor angiogenesis; Vascular endothelial growth factor

\section{INTRODUCTION}

Therapeutic options for patients with cancer, such as melanoma, prostate cancer, and renal cell carcinoma, have increased during the past decade because of improved understanding of tumor biology and availability of targeted agents. Vascular endothelial growth factor (VEGF) and related signaling pathways are considered critically important in the pathogenesis, growth, and development of cancer. Inhibiting the VEGF signaling pathway has been investigated as a therapeutic strategy for cancer, either by blockade of VEGF ligand binding or inhibition of the VEGF receptor (VEGFR) [1].

\section{Vascular Endothelial Growth Factor}

A prevailing hypothesis is that when a tumor outgrows its blood supply, the resulting hypoxia switches on hypoxia inducible factor (HIF) $\propto$ signaling that stimulates the production of several pro-angiogenic factors $[1,2]$. An important pro-angiogenic pathway is that mediated by the VEGF family of ligands, which includes six growth factors [VEGF-A, VEGF-B, VEGF-C, VEGF-D, VEGF-E, and placental growth factor (PlGF)] and three VEGF receptors [2-4]. The VEGFRs are transmembrane tyrosine kinases present mainly in endothelial cells. When circulating VEGFs bind to VEGFRs, signal transduction pathways are activated that may promote endothelial cell proliferation, differentiation, migration, and angiogenesis [3]. The VEGF/ VEGFR signaling pathway is involved in normal developmental and wound healing processes such as embryologic development, skeletal growth, and wound healing [4].

In addition, it has been shown that tumor angiogenesis is usually 'dysfunctional', with profound alterations, in terms of shape, size, and permeability, as compared to normal angiogenesis [5]. Anti-angiogenic treatments may lead to, not only inhibition of angiogenesis, but also normalization of such abnormal vasculature, allowing, for example, better extravasation of anticancer drugs in tissues. Such a phenomenon appears to be critical in colorectal cancer (CRC), where indeed anti-angiogenic treatments, such as bevacizumab, are commonly administered in an adjunction to cytotoxic chemotherapy [5].

Furthermore, VEGF expression in CRC may be associated with metastasis, recurrence, and poor prognosis [6-8]. Therefore, inhibitors of VEGF or VEGFRs were developed to treat malignancies by limiting tumor angiogenesis 
associated with activated VEGF/VEGFR pathways. Here, we review the rationale and clinical development of aflibercept for the treatment of cancer with particular emphasis on its use in CRC.

\section{METHODS}

A search of PubMed and MEDLINE from January 2000 to August 2013 was performed using the following terms (or combination of terms): VEGF, colorectal cancer, metastatic colorectal cancer (mCRC), aflibercept, ziv-aflibercept. Studies were limited to those published in English.

We also reviewed reference lists and discussed search results with experts. Due to its novel nature, abstracts or presentations of any national or international conference on aflibercept were assessed for inclusion.

\section{VEGF-BINDING AGENTS}

\section{Aflibercept (VEGF Trap)}

Aflibercept, also known as ziv-aflibercept or VEGF Trap $\quad$ (Zaltrap $^{\circledR}$, Regeneron, Pharmaceuticals, Tarrytown, NY, USA and Sanofi, Bridgewater, NJ, USA), has been approved by the US Food and Drug Administration for the treatment of macular degeneration and mCRC [9-11]. Aflibercept is a fusion compound that includes human VEGFR1 (FLT1), extracellular immunoglobulin (Ig) domain number 2, and VEGFR2 (KDR) extracellular Ig domain number 3, fused to human IgG1 Fc (Fig. 1) [12]. This fusion protein acts as a soluble decoy receptor that binds ("traps") VEGF and prevents VEGF

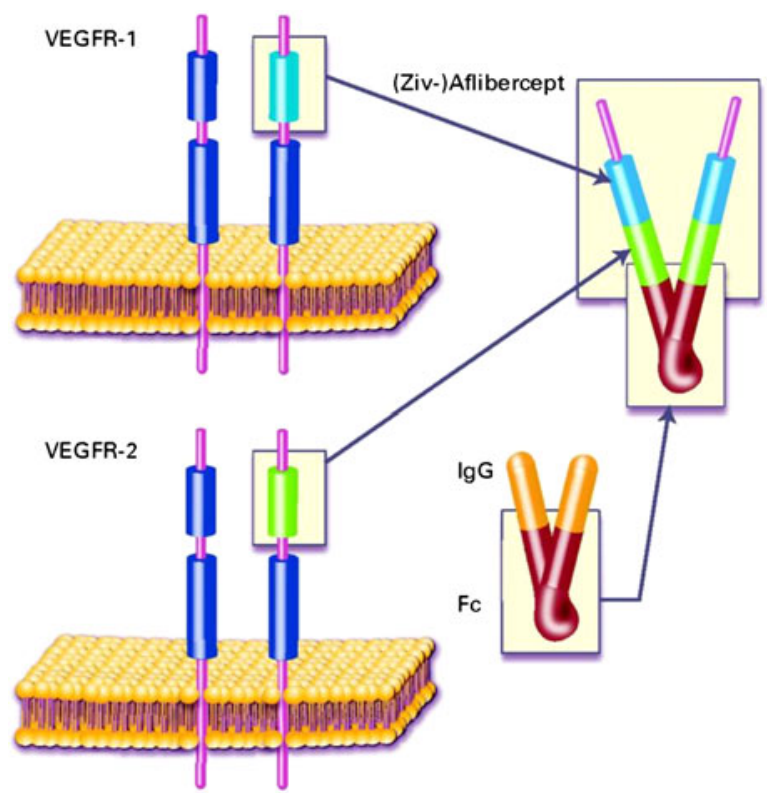

Fig. 1 Schematic structure of aflibercept, also known as ziv-aflibercept or VEGF trap. This drug is a fusion compound that includes human VEGFR1 (FLT1), extracellular Ig domain number 2, and VEGFR2 (KDR) extracellular Ig domain number 3, fused to the Fc region of a human IgG1 molecule. $\operatorname{Ig} G$ immunoglobulin G, $V E G F$ vascular endothelial growth factor, $V E G F R$ vascular endothelial growth factor receptor. Reproduced with permission from Ramlau et al. [12]

binding and signaling. Aflibercept binds to VEGF with high affinity $(\mathrm{Kd} \cong 1 \mathrm{pmol} / \mathrm{L})$ and also binds to PIFGs [13, 14].

Some tumors may become resistant to antiVEGF therapies because of an increase in the levels of PIGF [13]; therefore, it is feasible that the binding of aflibercept to PIGF could be of interest because by binding or "trapping" both VEGF and PIGF aflibercept could possibly overcome the development of resistance to other drugs such as bevacizumab [14]. In preclinical studies, aflibercept prevented the growth of micrometastases, caused regression and renormalization of vasculature in existing tumors, and inhibited new vascular growth [15, $16]$. 


\section{Aflibercept: Preclinical Data}

Aflibercept inhibits VEGF-induced phosphorylation of VEGFR-2 in human umbilical vein endothelial cells and VEGFinduced fibroblast proliferation in vitro [13]. In mice, aflibercept induces regression of capillaries, cessation of blood flow, and apoptosis of endothelial cells, and empty basement membrane sleeves [17, 18]. Several studies of the effect of aflibercept on tumor xenografts, including neuroblastoma, Wilms' tumor, ovarian cancer, pancreatic tumors, renal cell carcinoma, glioma, and CRC, have reported anti-angiogenic activity, including regression of blood vessels, decreased tumor vessel density and perfusion, inhibition of metastases, and improved survival [19]. Inhibition of tumor growth and improved survival were demonstrated for several animal tumor xenografts when aflibercept was administered with trastuzumab, docetaxel, paclitaxel, irinotecan, 5-fluorouracil, or radiotherapy [20-25].

\section{Clinical Data}

\section{Phase 1 Trials}

Several phase 1 studies of aflibercept, as a single agent or in combination with other drugs, were undertaken (Table 1) [26-34]. In an open-label, dose-escalation study, 38 patients with relapsed or refractory solid tumors initially received a single subcutaneous dose of aflibercept $25 \mu \mathrm{g} / \mathrm{kg}$ followed 4 weeks later by six weekly injections (escalating doses $0.025,0.05,0.1,0.2,0.4$, and $0.8 \mathrm{mg} / \mathrm{kg}$ ) or six twice-weekly injections $(0.8 \mathrm{mg} / \mathrm{kg})$ [30]. In this trial, no objective responses were observed, but 20 patients (53\%) had stable disease for $>1$ month and two patients had stable disease for $>1$ year. The most common adverse events (AEs) were proteinuria $(37 \%)$, fatigue (32\%), injection-site reaction $(18 \%)$, nausea $(17 \%)$, myalgia $(16 \%)$, anorexia (16\%), hypertension (13\%), and voice hoarseness (11\%). Grade 3/4 hypertension (8\% of patients) and proteinuria (3\% of patients) were reported [30].

In another trial evaluating the safety and efficacy of aflibercept, the agent was administered intravenously in doses ranging from 0.3 to $7.0 \mathrm{mg} / \mathrm{kg}$ every 2 weeks in 47 patients with refractory solid tumors or nonHodgkin's lymphoma [33]. A partial response [PR, defined by response evaluation criteria in solid tumors (RECIST) criteria] [35] was observed in three patients, and two patients had stable disease (SD). The most common toxicities observed were fatigue $(63.8 \%)$, nausea (36.2\%), and vomiting (27.7\%); grade 3 hypertension occurred $(16.7 \%$ patients at the $2.0 \mathrm{mg} / \mathrm{kg}$ dose; $42.9 \%$ patients at the $4.0 \mathrm{mg} / \mathrm{kg}$ dose; $75.0 \%$ patients at the $5.0 \mathrm{mg} / \mathrm{kg}$ dose) and grade 3 proteinuria was reported (1 patient, $7.0 \mathrm{mg} / \mathrm{kg}$ dose) (Table 1).

\section{Phase 2 Trials}

Several phase 2 clinical trials have assessed the safety and clinical activity of aflibercept, alone or in combination with cytotoxic drugs, in various solid tumors including CRC, non-small cell lung cancer (NSCLC), renal cell carcinoma, ovarian cancer, gynecologic cancer, soft tissue sarcoma, transitional cell carcinoma, melanoma, and glioblastoma (Table 2) [36-44].

Aflibercept was evaluated in two major phase 2 clinical trials for the treatment of mCRC [39, 44]. The first of these studies was a two-stage, multicenter, open-label, trial in 75 patients who had received prior treatment for the disease (Table 2) [39]. Patients were categorized into two groups dependent on previous treatment (prior bevacizumab, 51 patients; no prior bevacizumab, 24 patients), and all patients 


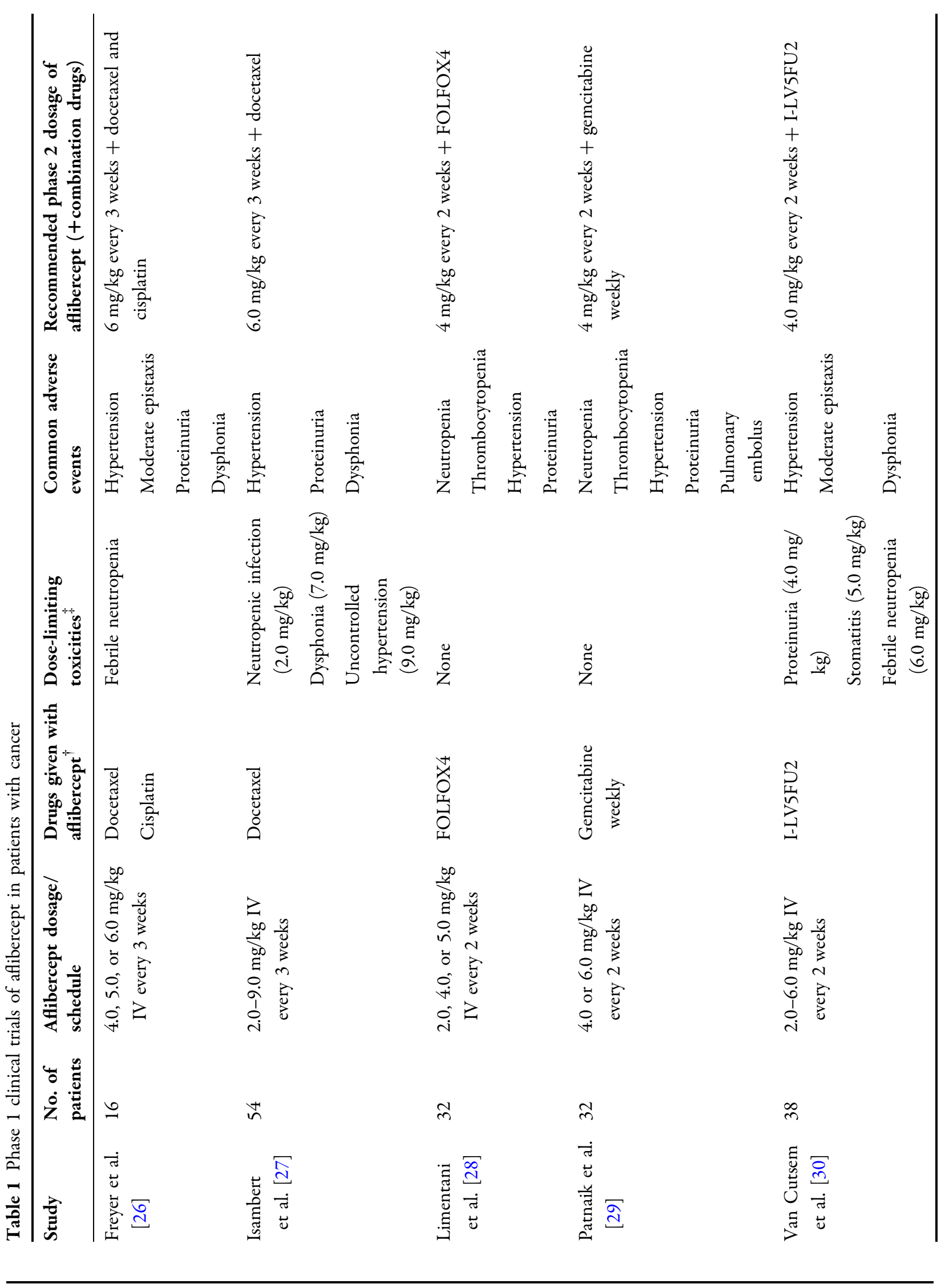




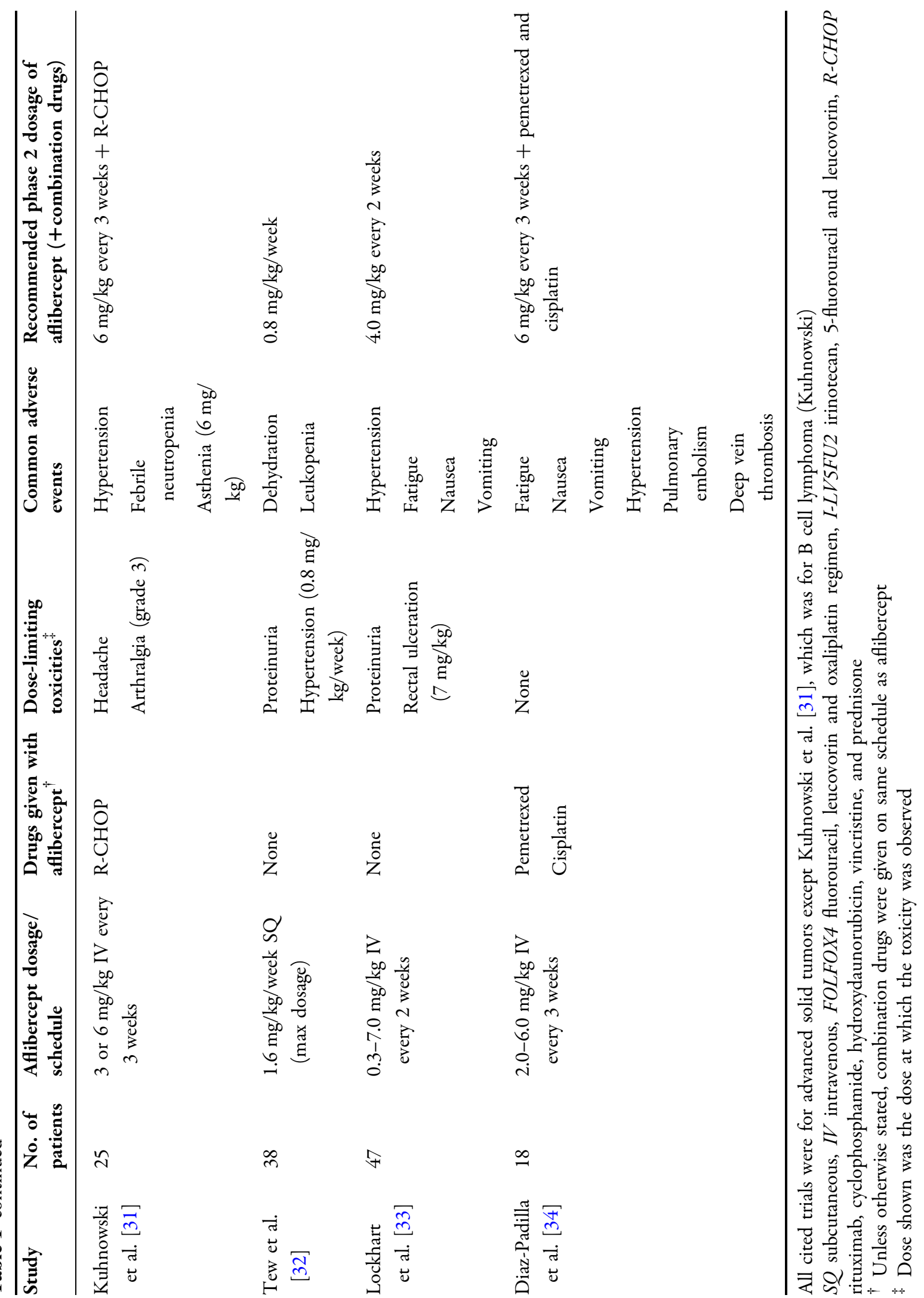




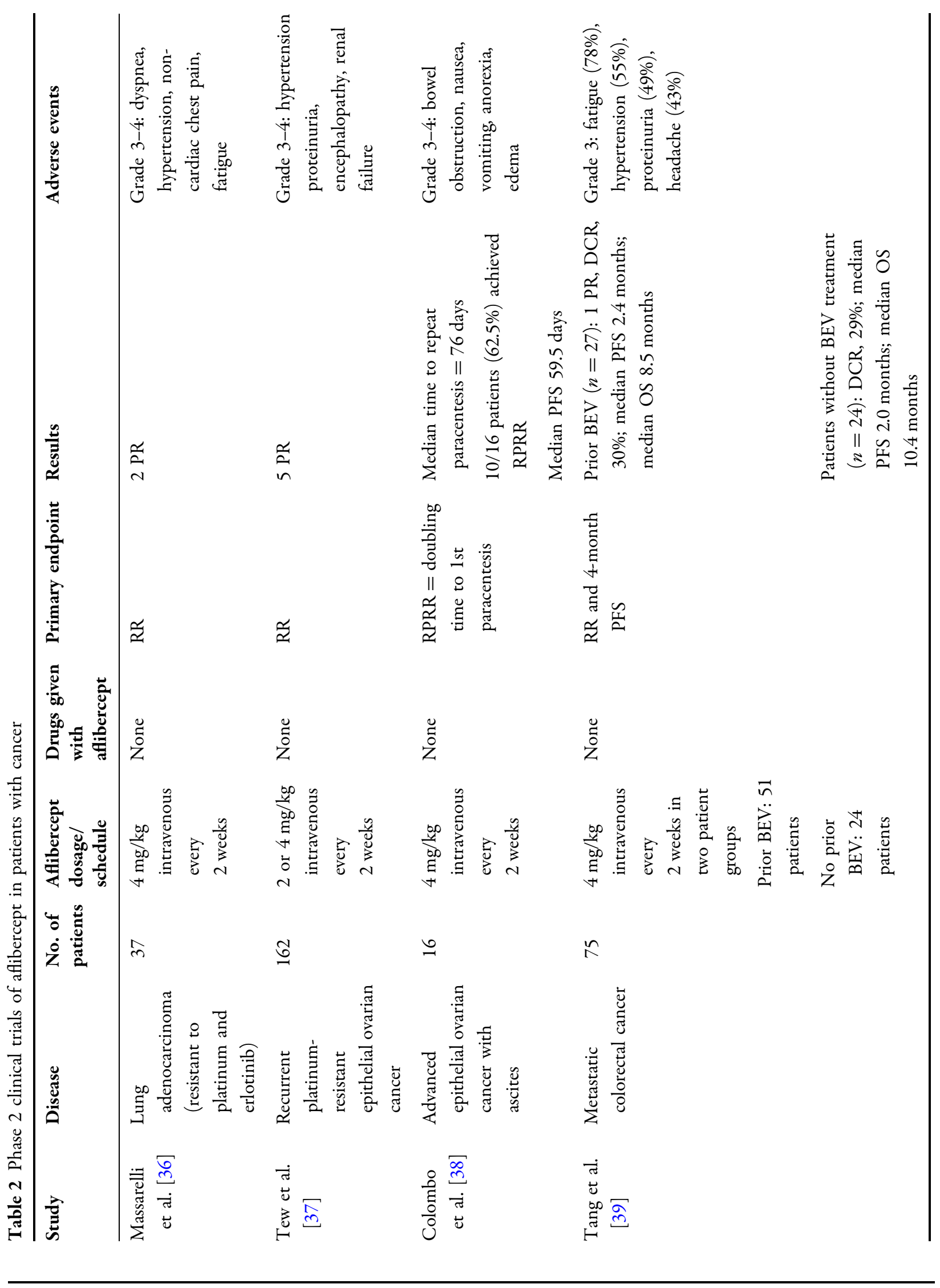




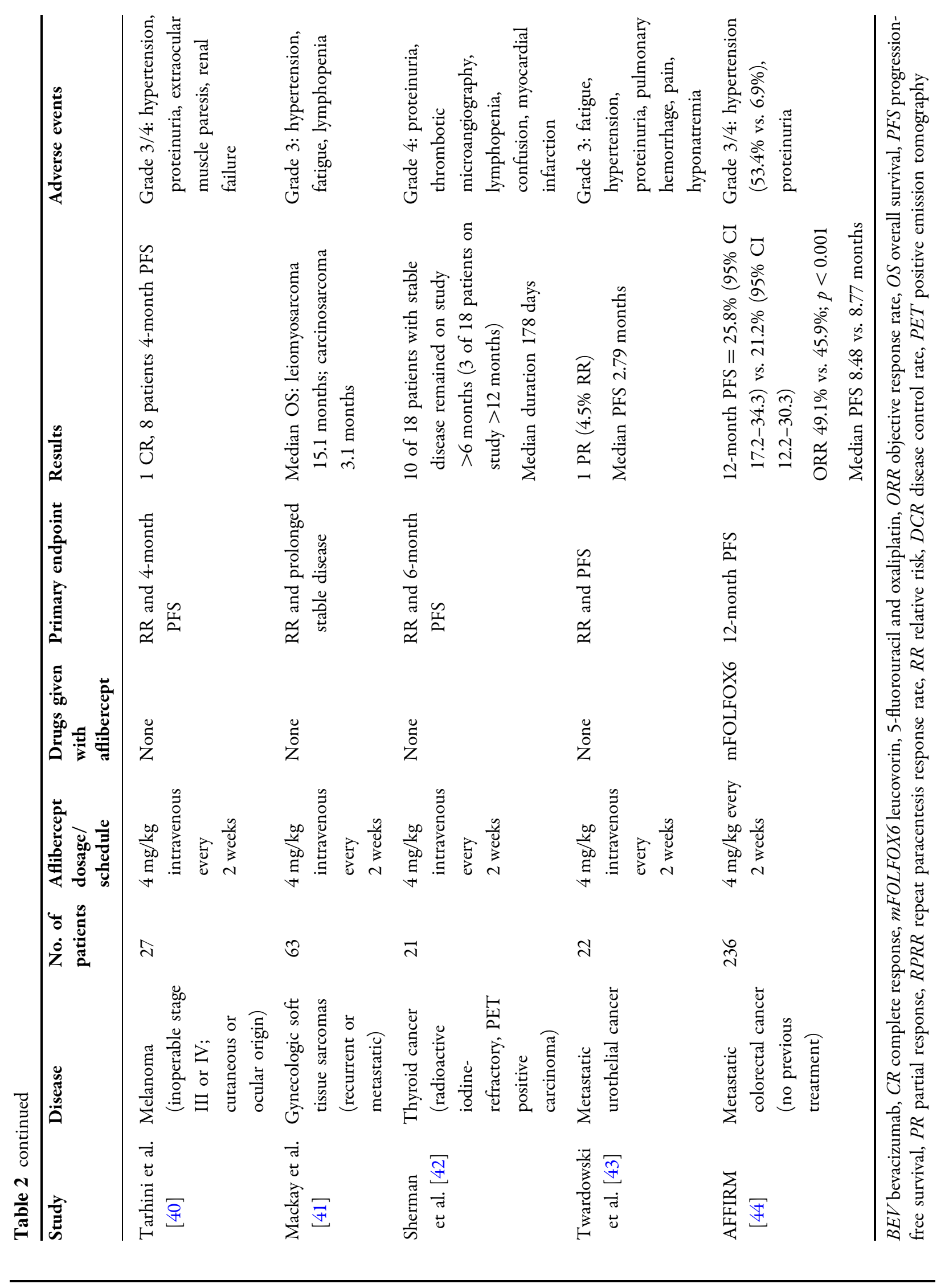


received aflibercept $(4 \mathrm{mg} / \mathrm{kg})$ intravenously every 2 weeks. After aflibercept treatment, the disease control rate (DCR, PR plus SD) and median PFS were similar in both groups (Table 2). AEs were common (Table 2). The authors concluded that aflibercept had modest activity against refractory or relapsed mCRC, regardless of prior therapy with bevacizumab.

The second major phase 2 trial was the AFFIRM trial which was a randomized openlabel trial evaluating the safety and efficacy of aflibercept and mFOLFOX6 (5-fluorouracil, leucovorin, oxaliplatin) as first-line treatment for mCRC [44]. Patients $(n=236)$ were randomized to aflibercept $(4 \mathrm{mg} / \mathrm{kg})$ plus mFOLFOX6 or mFOLFOX6 alone every 2 weeks (Table 2). Mean 12-month progression-free survival (PFS) was $25.8 \%$ for aflibercept plus mFOLFOX6 and $21.2 \%$ for mFOLFOX6 alone (Table 2). Addition of aflibercept to mFOLFOX6 significantly increased the objective response rate (ORR, $49.1 \%$ vs. $45.9 \%, p<0.001)$; however, median PFS was similar with or without aflibercept, and patients who received aflibercept had a higher frequency $(53.4 \%$ vs. $6.9 \%)$ of grade $3 / 4$ hypertension (Table 2).

\section{Phase 3 Trials}

To date, four large phase 3 clinical trials have been conducted to evaluate aflibercept for the treatment of various cancers (Table 3) [12, 4547]. The VELOUR trial evaluated the efficacy and safety of adding aflibercept to FOLFIRI in patients with mCRC who had progressed after previous treatment with oxaliplatin-based chemotherapy [45]. The primary endpoint of the study was OS. The trial included 1,226 patients (18 countries) with measurable or non-measurable disease according to RECIST criteria, and a Karnofsky performance status of $\geq 70 \%$. Patients were randomized to receive treatment with FOLFIRI plus placebo or FOLFIRI plus aflibercept (Table 2). Addition of aflibercept resulted in improved OS [median OS 13.5 vs. 12.1 months; hazard ratio (HR) 0.817; $p=0032]$. Addition of aflibercept significantly improved PFS by 2.2 months (median PFS 6.9 vs. 4.7 months; HR 0.76; $p<0.0001)$ and ORR $(19.8 \%$ vs. $11.2 \%$; $p=0.0001)$. Pre-specified subgroup analysis based on prior bevacizumab exposure was comparable between patients who had or had not received bevacizumab. Based on this trial, the US Food and Drug Administration approved aflibercept for use with FOLFIRI as second-line treatment for patients with CRC who had progressed despite oxaliplatincontaining chemotherapy.

It is noteworthy that two recent phase 3 trials evaluating the addition of bevacizumab to chemotherapy after first progression in patients with mCRC (Table 4) [48, 49] reported findings comparable, in terms of response rate, DCR and survival, to the results of the VELOUR trial.

In the ML18147 study, patients, depending on the first-line therapy, received either oxaliplatin-based or irinotecan-based secondline chemotherapy. Median PFS was 5.7 vs. 4.1 months $(p<0.0001)$ and median OS was 11.2 vs. 9.8 months $(p=0.0062)$ in favor of the bevacizumab group [48]. The BEBYP trial was a phase 3 study that randomized patients with unresectable mCRC treated with bevacizumab plus first-line chemotherapy (fluoropyrimidine, FOLFIRI, FOLFOX or FOLFOXIRI) to receive a second-line chemotherapy with FOLFOX or FOLFIRI (depending on first-line chemotherapy) alone or in combination with bevacizumab at $5 \mathrm{mg} / \mathrm{kg}$ intravenously every 2 weeks. The study demonstrated an increased PFS by continuing bevacizumab in second-line (median PFS was 6.8 vs. 5.0 months, $p=0.0062$ ) [49]. 


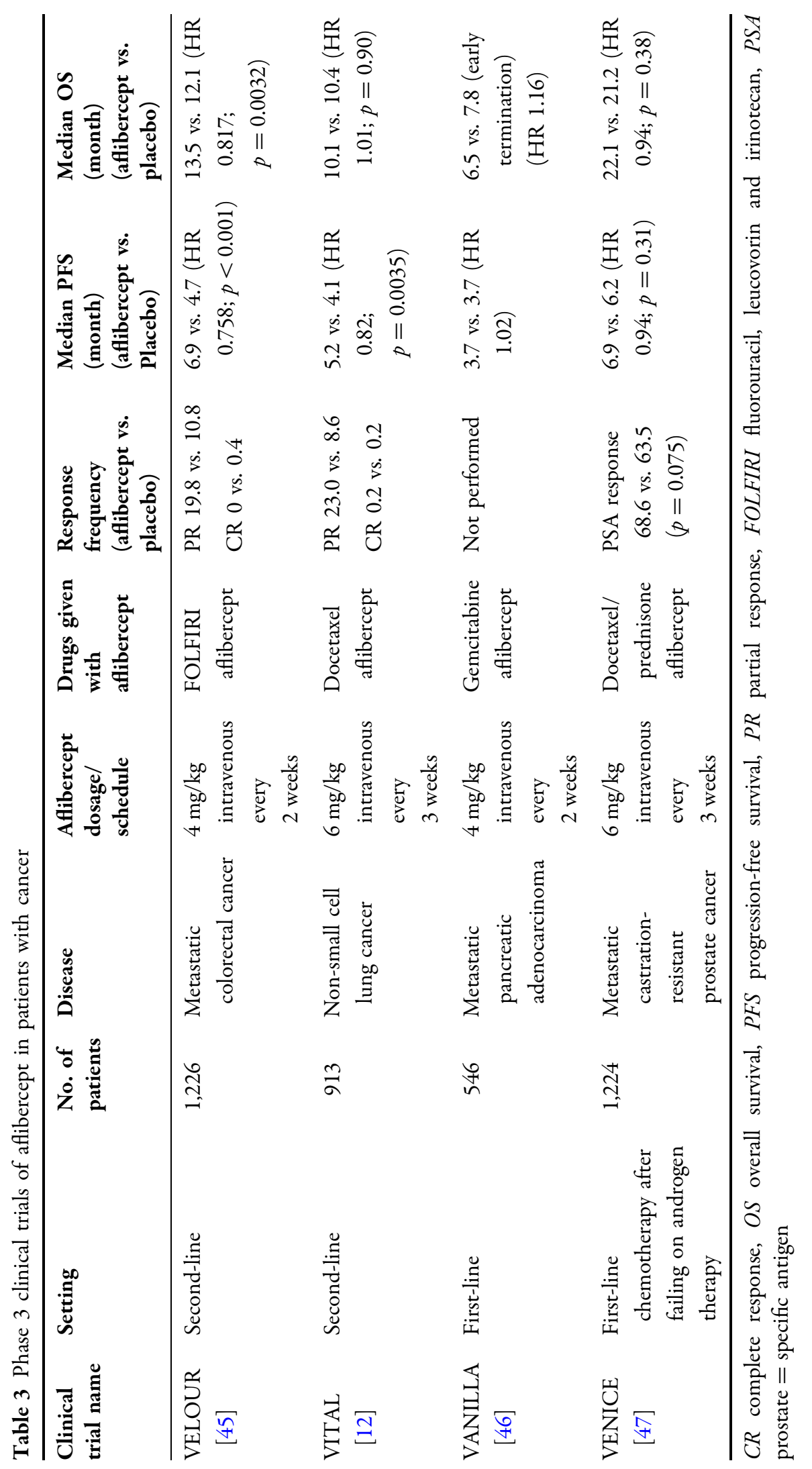




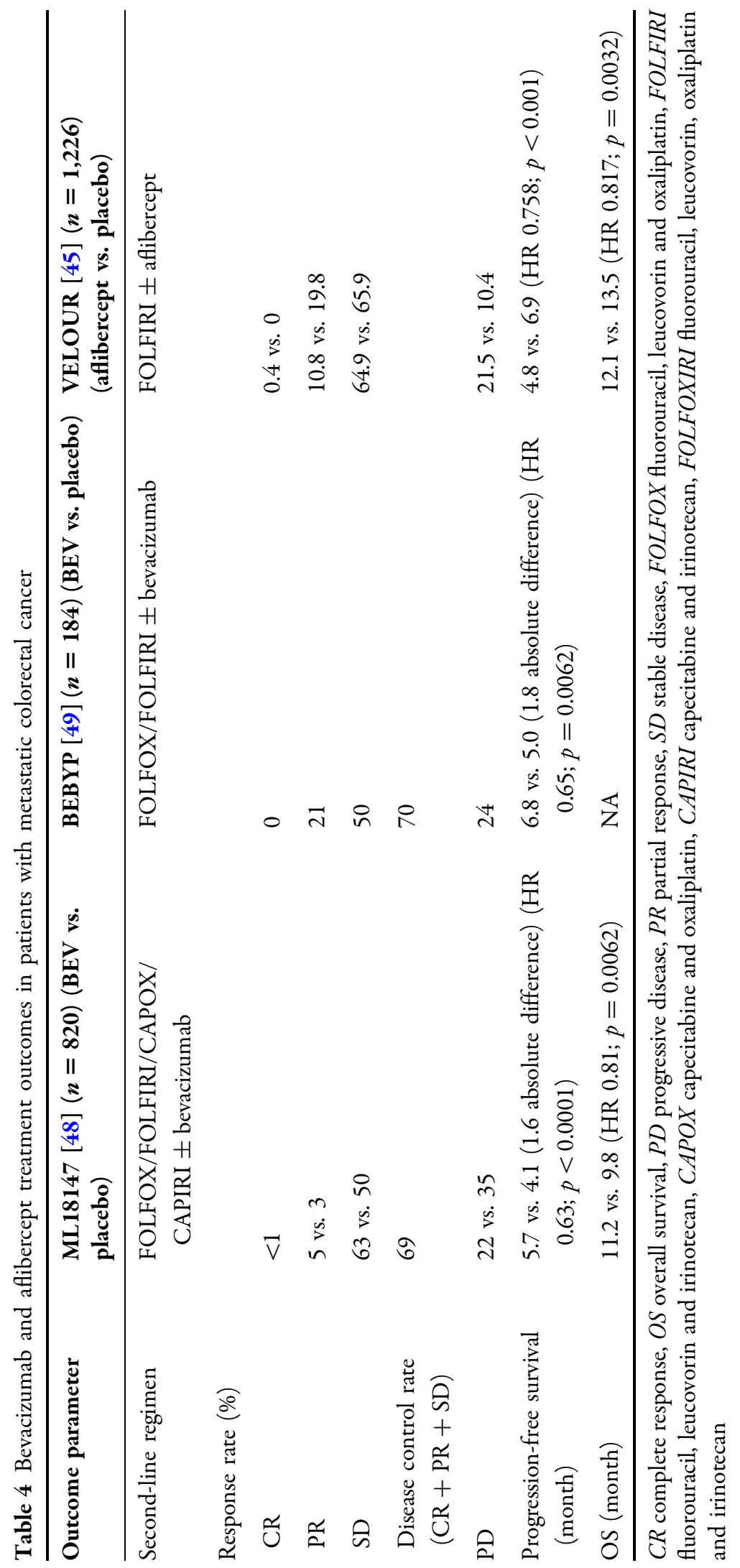




\section{VITAL Trial}

The VITAL trial was the second large randomized phase 3 trial to evaluate the efficacy and safety of aflibercept [12]. This was a double-blind, placebo-controlled study of aflibercept as second-line treatment for patients $(n=913)$ with NSCLC who had progressed after first-line platinum-based therapy (Table 3). OS was the primary endpoint. Patients received docetaxel plus aflibercept or docetaxel plus placebo. No difference was observed between the two treatment groups in terms of OS (median OS 10.1 vs. 10.4 months with/without aflibercept, respectively; HR 1.01; $p=0.90$ ) (Table 3 ). Median PFS was slightly prolonged in the aflibercept group (5.2 vs. 4.1 months; HR 0.82; $p=0.0035)$. ORR was also higher in patients who were treated with docetaxel plus aflibercept compared with patients treated with docetaxel plus placebo ( $23.2 \%$ vs. $8.8 \% ; p=0.001)$.

\section{VANILLA and VENICE Trials}

The remaining two phase 3 clinical trials of aflibercept were conducted in a first-line setting and in combination with chemotherapy. The VANILLA trial enrolled patients with metastatic pancreatic adenocarcinoma, while the VENICE trial assessed patients with metastatic castration-resistant prostate cancer [46, 47]. The VANILLA trial was terminated early because of an estimation of decreased survival in patients who received aflibercept vs. control patients (Table 3) [46]. The VENICE trial sought to discover an added benefit for the use of aflibercept in patients with metastatic androgen-independent prostate cancer [47]. The primary endpoint of the study was OS. A total of 1,224 patients were enrolled. Patients were randomized into $1: 1$ ratio to receive docetaxel/prednisone plus aflibercept or docetaxel/prednisone plus placebo. There was no statistically significant difference in OS between both groups (median OS 22.1 vs. 21.2 months, $p=0.38$; respectively); nor in PFS (6.9 vs. 6.2 months, respectively; $p=0.31$ ). However, patients in the aflibercept arm experienced a higher incidence of grade 3/4 AEs $(76.9 \%$ vs. $48.5 \%)$ which led to discontinuation rates of $43.9 \%$ for aflibercept vs. $20.9 \%$ in the placebo arm.

\section{Aflibercept: Adverse Events}

Adverse reactions to aflibercept were common in phase 1 and 2 trials (Tables 1, 2). In the phase 3 VELOUR trial, the tolerability profile for aflibercept plus FOLFIRI was consistent with that reported previously for aflibercept, FOLFIRI, and other anti-VEGF therapies. Aflibercept was associated with hypertension (19.1\%), proteinuria (7.9\%), and other common chemotherapy-related AEs such as diarrhea, hand-foot syndrome, and neutropenia [45]. In the phase 3 VITAL trial, patients who received aflibercept experienced a higher frequency of grade $3 / 4$ AEs including neutropenia (aflibercept $28.0 \%$, control $21.1 \%$ ); fatigue (aflibercept $11.1 \%$, control $4.2 \%$ ); stomatitis (aflibercept $8.8 \%$, control $0.7 \%$ ) and hypertension (aflibercept 7.3\%, control 0.9\%) [12].

In the VELOUR trial, the incidence of treatment-related AEs was similar among patients who received aflibercept with prior bevacizumab exposure (AEs, 100\% of patients) or no prior bevacizumab exposure (AEs, 98.9\% of patients). In the aflibercept component of the study, the frequency of grade 3/4 AEs was similar between patients who had received prior bevacizumab $(82.5 \%)$ and patients who had no prior bevacizumab experience (83.9\%) 
[45]. In both groups, $25 \%$ of aflibercept-treated patients discontinued treatment because of AEs. The most frequent AEs common to aflibercept and bevacizumab were grade $3 / 4$ neutropenia, diarrhea, asthenia, infections, and stomatitis; however, bevacizumab had a more favorable safety profile with less proteinuria and almost no gastrointestinal or constitutional toxicity.

The authors of a meta-analysis of three double-blind, placebo-controlled, phase 3 trials evaluating AEs associated with aflibercept concluded that the most common AEs associated with intravenous aflibercept were hypertension, venous and arterial thromboembolic events, hemorrhage, and proteinuria (Table 5) [50].

\section{BIOMARKERS}

Despite extensive efforts, researchers have yet to identify a reliable and reproducible biomarker that allows selection and identification of patients who are most likely to benefit from aflibercept therapy. Circulating biomarkers are currently under evaluation as potential tools to monitor treatment response to anti-VEGF drugs. For some cancers, response to treatment with aflibercept may be monitored by measuring levels of cytokines, growth factors, and myeloid biomarkers. In patients with glioblastoma, treatment with aflibercept was associated with decreased levels of VEGF and PIGF [51]. Lower levels of growth-regulated oncogene $\alpha$, hepatocyte growth factor, and stem cell growth factor $\beta$, and higher levels of free VEGF, were also associated with a PR in this patient group. Elevated baseline levels of CTACK/CCL27, MCP3/CCL7, MIF, and IP-10/ CXCL10, and a decrease in VEGFR1-positive monocytes from baseline to 24-h postaflibercept administration, have also been associated with improved response in glioblastoma patients [52].

Table 5 Adverse events associated with aflibercept in the treatment of cancer

\begin{tabular}{lcc}
\hline Adverse event $($ grade $>$ 3) & Aflibercept $(\boldsymbol{n}=\mathbf{1 , 3 3 3})$ & Placebo $(\boldsymbol{n}=\mathbf{1 , 3 2 9})$ \\
\hline Hypertension & 14.6 & 1.6 \\
Venous thromboembolic events & 6.4 & 6.1 \\
Hemorrhage & 1.5 & 3.1 \\
Arterial thromboembolic events & 1.0 & 1.7 \\
Proteinuria & 0.8 & 6.3 \\
Gastrointestinal perforation & 0.2 & 0.8 \\
Cardiac dysfunction & $<0.1$ & 0.5 \\
Fistula & $<0.1$ & 0.2 \\
Osteonecrosis & $<0.1$ & $<0.1$ \\
Impaired wound healing & 0 & 0.2
\end{tabular}

Data reported as percent patients. Data obtained from meta-analysis of three double-blind, placebo-controlled, phase 3 clinical trials with intravenous aflibercept [50]

Meta-analysis of total of 2,662 patients of three double-blind, placebo-controlled, phase 3 trials in patients with metastatic colorectal, lung and pancreatic cancer 


\section{DISCUSSION AND FUTURE DIRECTIONS}

Over the last decade there have been unprecedented advances in the treatment of mCRC. In the modern era, the average median survival duration has doubled, and patients usually live longer than 2 years largely due to the availability of new active agents. Last year alone in 2012, the FDA has approved two new drugs, aflibercept and regorafenib, for the treatment of mCRC due to demonstrated overall survival benefits.

Although, as shown in this review, aflibercept has demonstrated overall survival benefits when combined to FOLFIRI for the treatment of patients with mCRC that is resistant to or has progressed following an oxaliplatin-containing regimen, due to the lack of head to head trials, its place in the treatment paradigm of mCRC, with the availability of similar agents such as bevacizumab, is still not well defined. Similarly, the best way to combine and sequence all of these drugs to optimize treatment has not yet been established. In some cases, predictive biomarkers can influence the choice of treatment. For instance, patients who harbor a K-ras mutation may benefit from strategies targeting the EGFR [53]. Despite extensive research, there are no reliable or reproducible markers that predict response to anti-angiogenic therapy. These advances in therapy have not only resulted in nearly doubling of the median overall survival, but have also resulted in many challenges and raised several questions such as: why such agents have modest clinical success despite the promising preclinical data? Which VEGF inhibitor is more potent and better tolerated? Can these inhibitors be used in combination and if so, can this type of treatment be sustained? What are the future promising agents targeting VEGF and related signaling pathway and strategies to combat mCRC? How do these new agents such as aflibercept compare directly to the older medications such as bevacizumab and how can we select the proper agent between the currently available drugs?

Additionally, the challenge is how to determine the optimal sequencing of these agents in treatment naive patients, relapsed patients or in the maintenance setting. Lastly and perhaps most importantly, why aflibercept has failed to demonstrate similar benefits in other tumor types such as patients with NSCLC, prostate or pancreatic cancer and how we can improve patient selection and distinguish patients who may benefit best from aflibercept from those who will require other antiangiogenic agents.

\section{ACKNOWLEDGMENTS}

No funding or sponsorship was received for this study or publication of this article.

Conflict of interest. Dr. Salem and Dr. ElRefai declare no conflict of interest.

Compliance with ethics guidelines. This article does not contain any studies with human or animal subjects performed by any of the authors.

Open Access. This article is distributed under the terms of the Creative Commons Attribution Noncommercial License which permits any noncommercial use, distribution, and reproduction in any medium, provided the original author(s) and the source are credited. 


\section{REFERENCES}

1. Cook KM, Figg WD. Angiogenesis inhibitors: current strategies and future prospects. CA Cancer J Clin. 2010;60(4):222-43.

2. Tonini T, Rossi F, Claudio PP. Molecular basis of angiogenesis and cancer. Oncogene. 2003;22: 6549-56.

3. Hicklin DJ, Ellis LM. Role of the vascular endothelial growth factor pathway in tumor growth and angiogenesis. J Clin Oncol. 2005;23: 1011-27.

4. Galiano RD, Tepper OM, Pelo CR, et al. Topical vascular endothelial growth factor accelerates diabetic wound healing through increased angiogenesis and by mobilizing and recruiting bone marrow-derived cells. Am J Pathol. 2004;164: 1935-47.

5. Papetti M, Herman IM. Mechanisms of normal and tumor-derived angiogenesis. Am J Physiol Cell Physiol. 2002;282:C947-70.

6. Takahashi Y, Kitadai Y, Bucana CD, Cleary KR, Ellis LM. Expression of vascular endothelial growth factor and its receptor, KDR, correlates with vascularity, metastasis, and proliferation of human colon cancer. Cancer Res. 1995;55:3964-8.

7. Takahashi Y, Tucker SL, Kitadai Y, et al. Vessel counts and expression of vascular endothelial growth factor as prognostic factors in nodenegative colon cancer. Arch Surg. 1997;132:541-6.

8. Cascinu S, Staccioli MP, Gasparini G, et al. Expression of vascular endothelial growth factor can predict event-free survival in stage II colon cancer. Clin Cancer Res. 2000;6:2803-7.

9. United States Food and Drug Administration. FDA approves Eylea for eye disorder in older people. http://www.fda.gov/NewsEvents/Newsroom/Press Announcements/ucm280601.htm. Updated Nov 18, 2011. Accessed Feb 19, 2013.

10. United States Food and Drug Administration. Zivaflibercept. http://www.fda.gov/Drugs/Information OnDrugs/ApprovedDrugs/ucm314438.htm. Updated Aug 3, 2012. Accessed Feb 19, 2013.

11. United States Food and Drug Administration. FDA approves Zaltrap for metastatic colorectal cancer. http://www.fda.gov/NewsEvents/Newsroom/

PressAnnouncements/ucm314372.htm. Accessed Feb 18, 2013.

12. Ramlau R, Gorbunova V, Ciuleanu TE, et al. Aflibercept and docetaxel versus docetaxel alone after platinum failure in patients with advanced or metastatic non-small-cell lung cancer: a randomized, controlled phase III trial. J Clin Oncol. 2012;30:3640-7.

13. Fischer C, Jonckx B, Mazzone M, et al. Anti-PLGF inhibits growth of $\mathrm{VEGF}^{\circledR}$-inhibitor-resistant tumors without affecting healthy vessels. Cell. 2007;131(3):463-75.

14. Holash J, Davis S, Papadopoulos N, et al. VEGFTrap: a VEGF blocker with potent antitumor effects. Proc Natl Acad Sci USA. 2002;99:11393-8.

15. Huang J, Frischer JS, Serur A, et al. Regression of established tumors and metastases by potent vascular endothelial growth factor blockade. Proc Natl Acad Sci USA. 2003;100:7785-90.

16. Byrne AT, Ross L, Holash J, et al. Vascular endothelial growth factor-trap decreases tumor burden, inhibits ascites, and causes dramatic vascular remodeling in an ovarian cancer model. Clin Cancer Res. 2003;9:5721-8.

17. Baffert F, Le T, Sennino B, et al. Cellular changes in normal blood capillaries undergoing regression after inhibition of VEGF signaling. Am J Physiol Heart Circ Physiol. 2006;290:H547-59.

18. Williams JL, Cartland D, Rudge JS, Egginton S. VEGF trap abolishes shear stress- and overloaddependent angiogenesis in skeletal muscle. Microcirculation. 2006;13:499-509.

19. Gaya A, Tse V. A preclinical and clinical review of aflibercept for the management of cancer. Cancer Treat Rev. 2012;38:484-93.

20. Le XF, Mao W, Lu C, et al. Specific blockade of VEGF and HER2 pathways results in greater growth inhibition of breast cancer xenografts that overexpress HER2. Cell Cycle. 2008;7:3747-58.

21. Hu L, Hofmann J, Holash J, et al. Vascular endothelial growth factor trap combined with paclitaxel strikingly inhibits tumor and ascites, prolonging survival in a human ovarian cancer model. Clin Cancer Res. 2005;11(19 Pt. 1):6966-71.

22. Lejeune $\mathrm{P}$, Chiron $\mathrm{M}$, Le Moigne $\mathrm{R}$, et al. Combination of the antiangiogenic agent aflibercept results in greater antitumor activity. In: Proceedings from the 99th American association for cancer research annual meeting. April 12-16, 2008; San Diego. Abstract 1107.

23. Abrahams C, Li B, Parveen A, Thurston G. Combination of aflibercept (VEGF trap) and docetaxel produces increased anti-tumor effects associated with enhanced changes to tumor vasculature. In: Proceedings from the 101st 
American association for cancer research annual meeting. April 17-21, 2010; Washington, DC. Abstract 5427.

24. Wachsberger PR, Burd R, Cardi C, et al. VEGF trap in combination with radiotherapy improves tumor control in u87 glioblastoma. Int J Radiat Oncol Biol Phys. 2007;67:1526-37.

25. Chiron M, Vrignaud $P$, Lejeune $P$, et al. Synergistic activity of aflibercept (VEGF trap) in combination with 5-fluorouracil and irinotecan in preclinical tumor models. In: Proceedings from the AACRNCIEORTC: molecular targets and cancer therapeutics. October 22-26, 2007; San Francisco. Abstract A13.

26. Freyer G, Isambert N, You B, et al. Phase I doseescalation study of aflibercept in combination with docetaxel and cisplatin in patients with advanced solid tumors. Br J Cancer. 2012;107:598-603.

27. Isambert N, Freyer G, Zanetta S, et al. Phase I doseescalation study of intravenous aflibercept in combination with docetaxel in patients with advanced solid tumors. Clin Cancer Res. 2012;18:1743-50.

28. Limentani SA, Just R, Purdham A, et al. A phase I dose escalation and pharmacokinetic (PK) study of intravenous (iv) aflibercept (VEGF trap) plus FOLFOX4 in patients (pts) with advanced solid tumors: preliminary results [abstract]. J Clin Oncol. 2008;26(Suppl):3556.

29. Patnaik A, Pipas M, Rosen L, et al. A phase I dose escalation and pharmacokinetic (PK) study of intravenous (iv) aflibercept (VEGF trap) plus weekly gemcitabine (Gem) in patients (pts) with advanced solid tumors: preliminary results [abstract]. J Clin Oncol. 2008;26(Suppl):3558.

30. Van Cutsem E, Khayat D, Verslyp C, et al. Phase I dose-escalation study of intravenous aflibercept administered in combination with irinotecan, 5-fluorouracil and leucovorin in patients with advanced solid tumors. Eur J Cancer. 2013;49: 17-24.

31. Kuhnowski F, Thieblemont C, Jardin F, et al. A phase I study of IV aflibercept (Afl) in combination with R-CHOP in untreated patients (pts) with B-cell lymphoma [abstract]. J Clin Oncol. 2010;28 (15S):8010.

32. Tew WP, Gordon M, Murren J, et al. Phase 1 study of aflibercept administered subcutaneously to patients with advanced solid tumors. Clin Cancer Res. 2010;16:358-66.

33. Lockhart AC, Rothenberg ML, Dupont J, et al. Phase I study of intravenous vascular endothelial growth factor trap, aflibercept, in patients with advanced solid tumors. J Clin Oncol. 2010;28:207-14.

34. Diaz-Padilla I, Siu LL, San Pedro-Salcedo M, et al. A phase I dose-escalation study of aflibercept administered in combination with pemetrexed and cisplatin in patients with advanced solid tumours. Br J Cancer. 2012;107:604-11.

35. Eisenhauer EA, Therasse P, Bogaerts J, et al. New response evaluation criteria in solid tumours: revised RECIST guideline (version 1.1). Eur J Cancer. 2009;45(2):228-47.

36. Massarelli E, Miller VA, Leighl NB, et al. Phase II study of the efficacy and safety of intravenous (IV) AVE0005 (VEGF trap) given every 2 weeks in patients (Pts) with platinum- and erlotinibresistant adenocarcinoma of the lung (NSCLA) [abstract]. J Clin Oncol. 2007;25(18S):7627.

37. Tew WP, Colombo N, Ray-Coquard I, et al. VEGFtrap for patients (pts) with recurrent platinumresistant epithelial ovarian cancer (EOC): preliminary results of a randomized, multicenter phase II study [abstract]. J Clin Oncol. 2007;25 (18S):5508.

38. Colombo N, Mangili G, Mammoliti S, et al. A phase II study of aflibercept in patients with advanced epithelial ovarian cancer and symptomatic malignant ascites. Gynecol Oncol. 2012;125:42-7.

39. Tang P, Cohen SJ, Kollmannsberger C, et al. Phase II clinical and pharmacokinetic study of aflibercept in patients with previously treated metastatic colorectal cancer. Clin Cancer Res. 2012;18: 6023-31.

40. Tarhini AA, Christensen S, Frankel P, et al. Phase II study of aflibercept (VEGF trap) in recurrent inoperable stage III or stage IV melanoma of cutaneous or ocular origin [abstract]. J Clin Oncol. 2009;27(15S):9028.

41. Mackay HJ, Buckanovich RJ, Hirte H, et al. A phase II study single agent of aflibercept (VEGF trap) in patients with recurrent or metastatic gynecologic carcinosarcomas and uterine leiomyosarcoma. A trial of the Princess Margaret Hospital, Chicago and California Cancer Phase II Consortia. Gynecol Oncol. 2012;125:136-40.

42. Sherman EJ, Ho AL, Haque S, et al. A phase II study of VEGF trap (aflibercept) in patients with radioactive iodine-refractory, positron emission tomography (PET) positive thyroid carcinoma [abstract]. J Clin Oncol. 2010;28(15S):5587.

43. Twardowski P, Stadler WM, Frankel P, et al. Phase II study of aflibercept (VEGF-trap) in patients with recurrent or metastatic urothelial cancer, a 
California cancer consortium trial. Urology. 2010;76:923-6.

44. Pericay CFG, Saunders M, Thomas A, et al. Phase 2 randomized, noncomparative open-label study of aflibercept and modified FOLFOX6 in the first line treatment of metastatic colorectal cancer (AFFIRM): abstract O-0024. Ann Oncol. 2012;23(Suppl 4): iv5-18.

45. Van Cutsem E, Tabernero J, Lakomy R, et al. Addition of aflibercept to fluorouracil, leucovorin, and irinotecan improves survival in a phase III randomized trial in patients with metastatic colorectal cancer previously treated with an oxaliplatin-based regimen. J Clin Oncol. 2012;30: 3499-506.

46. Riess H, Manges R, Karasek P, et al. Double-blind, placebo-controlled randomized phase III trial of aflibercept (A) plus gemcitabine $(G)$ versus placebo $(P)$ plus gemcitabine $(G)$ in patients with metastatic pancreatic cancer: final results. In: Proceedings from the 12th world congress on gastrointestinal cancer. June 30-July 3, 2010; Barcelona. Abstract O-0006.

47. Tannock IF, Fizazi K, Ivanov S, et al. Aflibercept versus placebo in combination with docetaxel and prednisone for treatment of men with metastatic castration-resistant prostate cancer (VENICE): a phase 3 , double-blind randomised trial. Lancet Oncol. 2013;14(8):760-8

48. Bennouna J, Sastre J, Arnold D, et al. Continuation of bevacizumab after first progression in metastatic colorectal cancer (ML18147): a randomized phase 3 trial. Lancet Oncol. 2013;14:29-37.

49. Gruppo Oncologico Nord Ovest. Continuation of bevacizumab beyond progression improves outcome in patients with metastatic colorectal cancer (BEBYP). Late breaking abstract \#17; ESMO 2012 congress of the European society for medical oncology in Vienna. http://abstracts.webges.com/ viewing/view.php?congress=esmo2012\&congress $\mathrm{id}=370$ \&publication_id=2734. Accessed Sept 25, 2013.

50. Allegra CJ, Tabernero J, Rougier P, et al. Metaanalysis of anti-VEGF class adverse events from three double-blind $(\mathrm{Db})$ placebo (Pbo)-controlled phase III trials with IV aflibercept (Afl). J Clin Oncol. 2012;(suppl 4: abstr 561).

51. Piao Y, Heymach JV, Bekele B, et al. Circulating cytokine and angiogenic factors as predictive biomarkers of glioblastoma response to aflibercept (VEGF trap) [abstract]. J Clin Oncol. 2009;27 (15S):2029.

52. de Groot JF, Piao Y, Tran H, et al. Myeloid biomarkers associated with glioblastoma response to anti-VEGF therapy with aflibercept. Clin Cancer Res. 2011;17:4872-81.

53. Karapetis CS, Khambata-Ford S, Jonker DJ, et al. K-ras mutations and benefit from cetuximab in advanced colorectal cancer. N Engl J Med. 2008; 359:1757-65. 